\title{
Massage Therapy in Patients With Cancer Pain: A Review on Palliative
}

\section{Care}

\author{
Mojtaba Miladinia, ${ }^{1}$ Shahram Baraz, ${ }^{1,}$ Kourosh Zarea, ${ }^{1}$ and Elham Mousavi Nouri ${ }^{1}$ \\ ${ }^{1}$ Nursing Care Research Center in Chronic Diseases, School of Nursing and Midwifery, Ahvaz Jundishapur University of Medical Sciences, Ahvaz, IR Iran \\ "Corresponding author: Shahram Baraz, Nursing Care Research Center in Chronic Diseases, School of Nursing and midwifery, Ahvaz Jundishapur University of Medical \\ Sciences, Ahvaz, Iran. Tel/Fax: +98-6133738333, E-mail: shahrambaraz@ajums.ac.ir
}

Received 2016 February 22; Revised 2016 July 26; Accepted 2016 August 10.

\begin{abstract}
Introduction: Cancer-related pain (CRP) and its treatments are common and the scariest problems that patients with cancer fear and negatively affect their quality of life. Despite medical intervention, the pain of cancer still remains a clinical problem. Thus, the use of complementary medicine methods such as massage therapy is essential to control pain in the patients.

Methodology: It was a review type study limited to national and international studies from 1995 to 2015 . Searching processes were completed by electronic databases and search engines. Finally, based on inclusion and exclusion criteria as well as the elimination of duplicate studies, nine articles were selected for final review among which five were clinical trials and four were review or metaanalysis articles.

Results: In all five clinical trials, massage therapy reduced pain of patients with cancer, which reflects the positive effects of massage therapy in adult patients with cancer. In addition, although various methods of massage therapy were employed, with short-term and long-term periods, it still had a positive impact. Meanwhile, four review or meta-analysis studies while different in the year of study, inclusion and exclusion criteria, manifested that the results of massage therapy was an effective non-pharmacological pain control in patients with cancer.

Conclusions: Finally, it can be concluded that massage therapy is an effective non-pharmacological way to control pain in adult patients with cancer. Furthermore, studies in Iran on the effects of massage therapy on pain in patients with cancer are limited and much more research is needed in this area.
\end{abstract}

Keywords: Massage Therapy, Pain, Cancer, Review Article

\section{Introduction}

Cancer is a chronic condition whose incidence is increasing throughout the world. Patients with cancer face many chronic problems caused by the disease and treatments. One of the most important, widespread, and frightening problems in patients with cancer is chronic pain (1).

\subsection{Cancer-Related Pain}

Almost $30 \%$ of patients with cancer experience pain in diagnosis. The pain increases $65 \%-85 \%$ as the disease progresses. Moreover, $90 \%$ of the hospitalized patients experience pain (2). The pain inflicted by cancer comes from different reasons such as the growth and spread of tumors, the side effects of treatments such as chemotherapy, radiotherapy, surgery, and underlying medical conditions (3).

Pain increases mental disorders, sleep disorders, insolvency, hopelessness, and fear in patient and family and also causes a complicated process of physical, mental and emotional struggles. Pain also influences social relationships, daily routines, and working ability resulting in financial problems (4-6). It decreases different aspects of the quality of life in both patient and family (7-9). The timely and proper pain relief leads to faster recovery, increased satisfaction and the quality of a patient's life (10).

Nowadays some developments are made to lessen the pain through the pharmaceutical guides and frequent use of opioids for pain control in patients with cancer. However, almost $45 \%$ of such patients still report the lack of efficacy and side effects of medicinal treatments (11). In addition, more than $20 \%$ of patients resist against medicines, and those who do not resist face the side effects of opioids. Such unwanted effects include respiratory depression, sleep disorders, nausea and vomiting, constipation, itching, addiction, etc. This problem is so serious that some patients prefer to endure the pain rather than to use opioids. On one hand, such drugs are riskier in older patients (12). On the other hand, the pain caused by cancer still remains a clinical problem in such patients (13); therefore, it is essential to employ non-pharmacological methods or the complementary medicine therapies such as massage therapy to control the pain in such patient (11). 


\subsection{Massage Therapy}

Massage therapy dates back to thousands of years ago. One of the nursing duties, the most common one, is to provide the most effective and safest complementary medicine therapies throughout the world $(14,15)$. Massage therapy is a physical and scientific manipulation with different intensities applied at a specific speed and harmony in direct relation to body tissues for rehabilitation, health promotion, and feeling good $(16,17)$. Massage therapy results in physiological effects (increased parasympathetic activities, increased blood flow to muscles and skin, increased relaxation hormone, and reduced stress hormone), psychological effects (relaxation and reduced anxiety), neurological effects (reduced spasticity and muscle strain, and reduced neuromuscular stimulation) and biomechanical effects (increased joint mobility, reduced active and passive stiffness, and increased muscle compliance), and it can control pain (17). Therefore, massage therapy can be used along with medicinal methods in patients with cancer.

\subsection{Context}

Currently, different methods of complementary medicine, especially massage therapy, are not used enough in Iran. One of the main reasons is that few studies are conducted on the use of complementary medicine. On the other hand, there are some problems with using pharmaceutical methods in patients with cancer in Iran. These problems make non-pharmaceutical methods more essential. The results of a study indicated that the prescription and consumption of opioids in patients with cancer would not follow a known and logical procedure in Iran, and frequent failures are observed in the prescription of medicine (18). Obviously, if simpler and safer methods are available to control pain in patients with cancer, it is not reasonable to deal with the abovementioned problems (3). Massage therapy can be considered an easy, safe, available and appropriate method. There are no review studies about massage therapy and cancer-related pain in Iran. Therefore this review study can help the healing teams to make better decisions in practice.

\section{Objectives}

The current study aimed to review domestic and foreign papers on the effect of massage therapy on pain in patients with cancer to obtain a more comprehensive perspective on the use of this non-pharmaceutical method in patients with cancer.

\section{Methods}

The review study included domestic and foreign research works from 1995 to 2015 . The initial search was conducted from December 2012 to April 2013. The search was updated in April 2015.

\subsection{Inclusion and Exclusion Criteria}

The inclusion criteria were as follows: 1) only review studies on meta-analyses, and clinical trials on massage therapy, 2) Studies on patients with cancer above 18 years old, 3) patients diagnosed with cancer (not a specific type of cancer), 4) pain studied as a variable, 5) Studies conducted in English and Farsi, and 6) studies conducted from 1995 onward. The exclusion criteria were as follows: 1) abstracts, 2) having no control groups, 3) using other methods of complementary medicine along with massage therapy, and 4) considering a specific type of cancer.

\subsection{Data Source and Study Selection}

Electronic databases and search engines such as MEDLINE, excerpta medica database (EMBASE), cumulative index of nursing and allied health literature (CINAHL), Islamic world science citation center (ISC), Google Scholar, international scientific indexing (ISI), alumni medical library (MEDLIB), Magiran, and scientific information databases (SID) were employed to conduct the search. The search terminologies were looked up in Farsi and English. The search terminologies included (cancer OR oncological OR chemotherapy) AND (pain OR pains OR palliative) AND (massage) AND (review article OR meta-analysis OR systematic review OR randomized clinical trial). Based on the search terminologies of 183 papers, nine papers were finally selected with respect to the inclusion and exclusion criteria after deleting repetitive papers. Among these nine papers, five were clinical trials and four were reviews or meta-analyses (Figure 1). Moreover, there were eight foreign papers and only one Iranian one. For the sake of final investigation, Table 1 indicates clinical trials, and Table 2 shows review articles and meta-analyses.

\section{Results}

\subsection{Randomized Clinical Trial Studies}

Among the five selected clinical trials, 250 adult patients with cancer were studied, and 162 individuals (64.8\%) received massage therapy in intervention group (Table 1 ). Four of the clinical trials included both male and female subjects $(19,20,22,23)$, and the subjects were all female in the study conducted by Nadjafi Ghezeljeh et al. (21). The number of males was greater than that of females only 
Table 1. Randomized Clinical Trial Studies

\begin{tabular}{|c|c|c|c|c|c|}
\hline Authors & Year & Place & Sample Size & Main Result & Signification \\
\hline Toth et al. (19) & 2013 & Israel & 39 & $\downarrow$ pain & NS \\
\hline Jane et al. (20) & 2011 & Taiwan & 72 & $\downarrow$ pain & Sig \\
\hline Nadjafi Ghezeljeh et al. (21) & 2003 & Iran & 75 & $\downarrow$ pain & Sig \\
\hline Wilkie et al. (22) & 2000 & USA & 29 & $\downarrow$ pain & Sig \\
\hline Ahles et al. (23) & 1999 & USA & 35 & $\downarrow$ pain & NS \\
\hline
\end{tabular}

Abbreviations: $\downarrow$, decrease; NS, not significant; Sig, statically significant.

Table 2. Review and Meta-Analysis Studies

\begin{tabular}{|c|c|c|c|c|}
\hline Authors & Year & Language & The Number of Articles Reviewed & Main Result \\
\hline Lee et al. (24) & 2015 & English & 12 & Benefit \\
\hline Falkensteiner et al. (25) & 2011 & English & 6 & Benefit \\
\hline Ernst (26) & 2009 & English & 14 & Benefit \\
\hline Jane et al. (27) & 2008 & English & 15 & Benefit \\
\hline
\end{tabular}

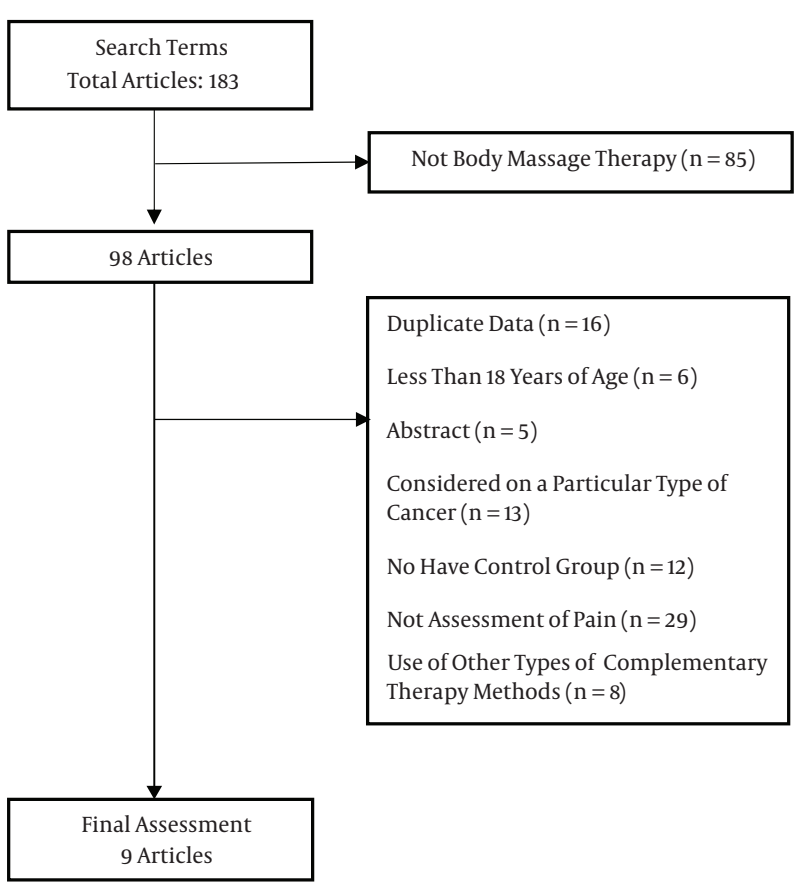

Figure 1. Flow Diagram of Study Inclusion and Exclusion

in one study (22). The intervention of massage therapy lessened patients' pain in all five clinical trials; however, there was a significant difference between the intervention group and control group with respect to the pain intensity only in three studies $(21,22,27)$. This indicated the positive effects of massage therapy on adult patients with cancer.

\subsection{Review and Meta-Analysis Studies}

In four of the studies, 47 clinical trials, reviews and meta-analyses were investigated in total (Table 2 ). The only meta-analysis was the study conducted by Lee et al. (24), and the rest of them were reviews (25-27). Although the year of study, inclusion and exclusion criteria, and the number of reviewed papers were different, the results of all four studies indicated that massage therapy could be useful as an effective non-pharmacological method to control pains in patients with cancer.

\section{Discussion}

Finally the results of the current review study showed that massage therapy could be useful as an effective nondrug method to control cancer-related pain.

In two studies conducted by Wilkie and Jane, the fullbody massage therapy was used. However, the massage sessions were different in the two studies in terms of duration and time. In Wilkie's study, there were two massage sessions in a week for several weeks; massage sessions were held for five days in Jane's study. However, the intensity of pain reduced in the intervention group in both studies (20, 22). Moreover, Swedish massage was used in two studies conducted by Toth and Ahles. The duration of massage and time of each session were also different in these two studies; however, the pain lessened after the therapy (19, 23). On the other hand, foot massage was used for a short period 
(three days) in Nadjafi Ghezeljeh et al. study (21). Considering the abovementioned points, it is noteworthy that although different methods of massage therapy were used in short and long periods of time, the therapy had positive effects. Therefore, different methods of massage therapy can be used to control the short-term and long-term pains in patients with cancer. In particular, short periods of massage therapy with lower costs can be used to control pain among patients with financial difficulties. However, Swedish massage was used in both Toth and Ahles's studies. Although pains lessened in the patients, there was no statistically significant difference between the massage group and control group, and more studies should be conducted on the use of this type of massage to control pain in patients with cancer $(19,23)$.

Although the average age ranged from 42 to 65 in these five clinical trials, the pain lessened in all of them, a fact which indicated that massage therapy could be used in adults at different ages. These studies were conducted in different countries; thus, various races, cultures and individual differences in samples could influence the perception of pain (9). Nevertheless, the positive effect of massage on pain relief was observed in these studies conducted in different parts of the world. In fact, massage therapy can be used to control pain in patients with cancer from different races.

One of the most important challenges observed in the results of the current review study was that there was only one study (conducted by Nadjafi Ghezeljeh et al.) which used massage therapy to control pain in patients with cancer in Iran. The study was conducted only on female patients (21). Perhaps, this is one of the reasons for the lack of controlling pain in patients with cancer in Iran. Therefore, more studies should be conducted on different methods of massage therapy in different places to observe the effects of this method on controlling pain in patients with cancer in Iran and find effective methods. Another challenge was that in all of the five clinical trials massage therapy was applied to patients hospitalized at oncology centers, not at home(Table1). Therefore, a similar study should be conducted on home-care patients to clarify the effect of massage therapy at home more and ascertain whether it is more or less effective. This matter can influence the more appropriate use of this method of complementary method.

There was also another empty spot in the conducted searches, indicating that no Iranian review studies were available on the effect of massage therapy on pains in patients with cancer. Thus, review studies including Iranian papers should be conducted on the effect of massage therapy on the pain and other problems (fatigue, sleep disorders, stress and depression) in patients with cancer.
Since pain has an inverse relationship with the quality of life, which can be improved by controlling pain $(7,9)$, massage therapy can be used to control pain in patients with cancer to promote their quality of life (19). Pharmaceutical methods are effective to control pains in patients with cancer (apart from various side effects, fear of using drugs and misusing them). However, it takes nurses a great deal of time to apply pharmaceutical methods, and Iran is facing a serious shortage of nurses (28). On the other hand, it is possible that the patients resist against drugs. In such a case, either drugs lose effects to control the pain, or the dose has to be increased. The latter action would increase the side effects and result in more pain (29). Therefore, non-pharmaceutical methods such as massage therapy can be used along with pharmaceutical methods to control pain better and use pharmaceutical methods less. As a result, nurses spend less time applying drugs.

Massage therapy is an easy, cheap, non-aggressive and safe method with no side effects. It provides a patient with comfort and ease and promotes the quality of life $(16,30$ 32 ). Increasing trust and safety among patients is easily accepted by them (25), a fact which leads to more acceptances of therapies and cooperation on patients' side (33).

\subsection{Conclusions}

This review study had several strengths: 1) this study was the first review study to investigate the effect of massage therapy on cancer-related pain in Iran which considered both domestic and foreign papers; 2 ) the review considered both randomized clinical trials and also review and meta-analysis studies.

Two general conclusions can be made from this review study: 1) the research results indicated that massage therapy was an effective non-pharmaceutical method to control pain in adult patients with cancer. Despite the differences in the demographics of statistical populations and different massage methods, similar results were also observed regarding pain relief in all studies; 2 ) the results also indicated that there were few Iranian studies conducted on the effect of massage therapy to control pain in patients with cancer.

\subsection{The Application of Research Findings in Clinical Practices}

Medical teams can use the results of the current study to have a more extensive look at the use of massage therapy to control pain in patients with cancer. Finally, it is suggested to employ different methods of massage therapy on larger samples both at hospital and home in future studies. In addition, it is advised to conduct more review studies on the effect of massage therapy on other problems in patients with cancer (fatigue, sleep disorder, depression 
and stress). Given the shortage of clinical studies in Iran, such studies should be especially conducted in Iran; hence, health cares are based on clinical evidence.

\section{Footnotes}

Authors' Contribution: Shahram Baraz and Mojtaba Miladinia: study conception and design, analysis, interpretation of data, drafting the manuscript, critical revisions for important intellectual content; Elham Mosavi Nouri: study conception and design, and acquisition of data.

Financial Disclosure: There was no financial interest related to the material in the manuscript.

Funding/Support: The current study was supported by Ahvaz Jundishapur University of Medical Sciences.

\section{References}

1. Tsai HF, Chen YR, Chung MH, Liao YM, Chi MJ, Chang CC, et al. Effectiveness of music intervention in ameliorating cancer patients' anxiety, depression, pain, and fatigue: a meta-analysis. Cancer Nurs. 2014;37(6):E35-50. doi: 10.1097/NCC.0000000000000116. [PubMed: 24662723].

2. Eyigor S, Eyigor C, Uslu R. Assessment of pain, fatigue, sleep and quality of life (QoL) in elderly hospitalized cancer patients. Arch Gerontol Geriatr. 2010;51(3):e57-61. doi: 10.1016/j.archger.2009.11.018. [PubMed: 20044154].

3. Shaban M, Rasulzadeh N, Mehran A, Morad Alizadeh F. Effect of two non-pharmacological methods (muscle relaxation and music therapy) on the pain in cancer patients. Hayat J. 2006;12(3):63-72.

4. Swarm RA, Abernethy AP, Anghelescu DL, Benedetti C, Buga S, Cleeland C. Adult Cancer Pain. J Nat Comprehens Cancer Network. 2013;11(8):992-1022.

5. Ovayolu N, Ovayolu O, Serce S, Tuna D, Pirbudak Cocelli L, Sevinc A. Pain and quality of life in Turkish cancer patients. Nurs Health Sci. 2013;15(4):437-43. doi:10.1111/nhs.12047. [PubMed: 23480371].

6. Miladinia M, Baraz S, Zarea K. Controlling Acute Post-operative Pain in Iranian Children with using of Music Therapy. Int J Pediatr. 2016;4(5):1725-30.

7. Kwekkeboom KL, Abbott-Anderson K, Cherwin C, Roiland R, Serlin RC, Ward SE. Pilot randomized controlled trial of a patient-controlled cognitive-behavioral intervention for the pain, fatigue, and sleep disturbance symptom cluster in cancer. J Pain Symptom Manage. 2012;44(6):810-22. doi: 10.1016/j.jpainsymman.2011.12.281. [PubMed: 22771125].

8. Manoochehri H, Shirazi M, Tafreshi M, Zayeri F. Chronic Pain Management and Being Vulnerable in Older People: A Qualitative Study. Jundishapur J Chron Dis Care. 2014;3(4).

9. Miladinia M, Baraz S, Shariati A, Malehi AS, Amadzadeh A. Relationship Between Chronic Pain and Quality of Life in Patients With Acute Leukemia Undergoing Chemotherapy. Jundishapur J Chron Dis Care. 2015;4(3).

10. Bastani F, Khosravi M, Barimnejad L, Haghani H. The effect of Acupressure on Chemotherapy-Induced Nausea and Vomiting among School age Children with Acute Lymphoblastic Leukemia. Complement Med J Faculty Nurs Midwifery. 2011;1(1):1-11.

11. Mao-Ying QL, Cui KM, Liu Q, Dong ZQ, Wang W, Wang J, et al. Stage-dependent analgesia of electro-acupuncture in a mouse model of cutaneous cancer pain. Eur J Pain. 2006;10(8):689-94. doi: 10.1016/j.ejpain.2005.11.001. [PubMed: 16376128].
12. Vinjamury SP, Li JT, Hsiao E, Huang C, Hawk C, Miller J, et al. Effects of acupuncture for cancer pain and quality of life - a case series. Chin Med. 2013;8(1):15. doi: 10.1186/1749-8546-8-15. [PubMed: 23895044].

13. Valeberg BT, Kolstad E, Smastuen MC, Miaskowski C, Rustoen T. The PRO-SELF pain control program improves family caregivers' knowledge of cancer pain management. Cancer Nurs. 2013;36(6):429-35. doi: 10.1097/NCC.0b013e3182747bcf. [PubMed: 23154516].

14. Siegel R, Naishadham D, Jemal A. Cancer statistics, 2013. CA Cancer J Clin. 2013;63(1):11-30. doi: 10.3322/caac.21166. [PubMed: 23335087].

15. Field T. Massage therapy research review. Complement Ther Clin Pract. 2016;24:19-31. doi: 10.1016/j.ctcp.2016.04.005. [PubMed: 27502797].

16. Karagozoglu S, Kahve E. Effects of back massage on chemotherapyrelated fatigue and anxiety: supportive care and therapeutic touch in cancer nursing. Appl Nurs Res. 2013;26(4):210-7. doi: 10.1016/j.apnr.2013.07.002. [PubMed: 24055114].

17. Weerapong P, Hume PA, Kolt GS. The mechanisms of massage and effects on performance, muscle recovery and injury prevention. Sports Med. 2005;35(3):235-56. [PubMed: 15730338].

18. Mohagheghi M, Nahvijo A, Sedighi Z. Pain control in patient with cancerby opioid drugs Tehran university of medical science. Med School J. 2003;61(2):95-102.

19. Toth M, Marcantonio ER, Davis RB, Walton T, Kahn JR, Phillips RS. Massage therapy for patients with metastatic cancer: a pilot randomized controlled trial. J Altern Complement Med. 2013;19(7):650-6. doi: 10.1089/acm.2012.0466. [PubMed: 23368724].

20. Jane SW, Chen SL, Wilkie DJ, Lin YC, Foreman SW, Beaton RD, et al. Effects of massage on pain, mood status, relaxation, and sleep in Taiwanese patients with metastatic bone pain: a randomized clinical trial. Pain. 2011;152(10):2432-42. doi: 10.1016/j.pain.2011.06.021. [PubMed: 21802850].

21. Nadjafi Ghezeljeh T, Rahimiha F, Mohaddes Ardebili F, Hosseini F. Effect of Foot Massage on Relaxation and Pain Intensity of Cancer Patients. Iran J Nurs. 2002;15(32):75-82.

22. Wilkie DJ, Kampbell J, Cutshall S, Halabisky H, Harmon H, Johnson LP, et al. Effects of massage on pain intensity, analgesics and quality of life in patients with cancer pain: a pilot study of a randomized clinical trial conducted within hospice care delivery. Hosp J. 2000;15(3):31-53. [PubMed: 11315685].

23. Ahles TA, Tope DM, Pinkson B, Walch S, Hann D, Whedon M, et al. Massage therapy for patients undergoing autologous bone marrow transplantation. J Pain Symptom Manage. 1999;18(3):157-63. [PubMed: 10517036].

24. Lee SH, Kim JY, Yeo S, Kim SH, Lim S. Meta-Analysis of Massage Therapy on Cancer Pain. Integr Cancer Ther. 2015;14(4):297-304. doi: 10.1177/1534735415572885. [PubMed: 25784669].

25. Falkensteiner M, Mantovan F, Muller I, Them C. The use of massage therapy for reducing pain, anxiety, and depression in oncological palliative care patients: a narrative review of the literature. ISRN Nurs. 2011;2011:929868. doi: 10.5402/2011/929868. [PubMed: 22007330].

26. Ernst E. Massage therapy for cancer palliation and supportive care: a systematic review of randomised clinical trials. Support Care Cancer. 2009;17(4):333-7. doi: 10.1007/s00520-008-0569-z. [PubMed: 19148685].

27. Jane SW, Wilkie DJ, Gallucci BB, Beaton RD. Systematic review of massage intervention for adult patients with cancer: a methodological perspective. Cancer Nurs. 2008;31(6):E24-35. doi: 10.1097/01.NCC.0000339242.51291.85. [PubMed:18987505].

28. Zarea K, Negarandeh R, Dehghan-Nayeri N, Rezaei-Adaryani M. Nursing staff shortages and job satisfaction in Iran: Issues and challenges. Nurs Health Sci. 2009;11(3):326-31.

29. Jarden M, Moller T, Kjeldsen L, Birgens H, Christensen JF, Bang Christensen $\mathrm{K}$, et al. Patient Activation through Counseling and ExerciseAcute Leukemia (PACE-AL)-a randomized controlled trial. BMC Cancer. 2013;13:446. doi: 10.1186/1471-2407-13-446. [PubMed: 24083543]. 
30. Miladinia M, Baraz S, Mousavi Nouri E, Gholamzadeh Baeis M. Effects of Slow-stroke Back Massage on Chemotherapy-induced Nausea and Vomiting in the Pediatrics with Acute Leukemia: a Challenge of Controlling Symptoms. Int J Pediatr. 2015;3(6.2):1145-52.

31. Miladinia M, Fakharzadeh L, Zarea K, Mousavi Nouri E. Anxiety Control in the Iranian Children with Chronic Leukemia: Use of a Non-drug Method. Int J Pediatr. 2016;4(1):1225-31.

32. Miladinia M, Baraz S, Shariati A, Malehi AS. Effects of Slow-Stroke
Back Massage on Symptom Cluster in Adult Patients With Acute Leukemia: Supportive Care in Cancer Nursing. Cancer Nurs. 2016 doi: 10.1097/NCC.0000000000000353. [PubMed: 26925992].

33. Currin J, Meister EA. A hospital-based intervention using massage to reduce distress among oncology patients. Cancer Nurs 2008;31(3):214-21. doi: 10.1097/01.NCC.0000305725.65345.f3. [PubMed: 18453878]. 